\title{
Can DIPPR Database be Used for an Estimation of the Speed of Sound? A Case Study of Liquid Hydrocarbons
}

\author{
Eugene B. POSTNIKOV \\ Theoretical Physics Department \\ Kursk State University \\ Radishcheva st. 33, Kursk 305000, Russia; e-mail: postnicov@gmail.com
}

(received April 11, 2016; accepted July 15, 2016)

\begin{abstract}
The paper analyses a possibility of utilising the information which is contained in DIPPR database for a calculation of the speed of sound, which is absent there. As an example, liquid hydrocarbons are considered: n-hexane, 1-hexene, cyclohexane, cyclohexene, benzene, and 1-hexanols, as well as representatives of $\mathrm{n}$-alkanes with various hydrocarbon chain lengths. It is shown that the Brelvi-O'Connell correlation for the reduced bulk modulus, supplied with the correlations for the internal pressure at the normal boiling temperature, results in the values having accuracy comparable with other DIPPR data for the region below the boiling point bounded by the values of the reduced density around $\rho_{r} \approx 3.5$. The source of errors originated from the Brelvi-O'Connell correlation for larger reduced densities is discussed.
\end{abstract}

Keywords: speed of sound; DIPPR; reduced bulk modulus; internal pressure.

\section{Introduction}

The DIPPR database (DIPPR) (The Design Institute for Physical Properties) is an extensive source for physical data on more than two thousand industrially important pure chemical compounds. It represents rigorously evaluated results of experimental measurements as well as high accuracy correlations for their calculations as functions of the temperature at saturation conditions. However, the speed of sound is not represented among the variety of the thermodynamic quantities provided there.

At the same time, the richness of the variety of compounds presented in DIPPR, as well as a wide applicability of DIPPR temperature-dependent equations for various industrial applications, e.g. (AspenPlus), see also (SANDLER, 2015) induce the questions: could one use DIPPR-available thermodynamic data for the determination of the estimated values of the speed of sound along the saturation curve, and what the accuracy of derivations and approximations used for this goal is.

The well-known thermodynamic equalities allow the expression of the speed of sound as

$$
c=\left(\beta_{T} \rho-\frac{T \alpha_{P}^{2}}{C_{p}}\right)^{-1 / 2}
$$

where the isobaric coefficient of the thermal expansion $\alpha_{P}$ and the isobaric heat capacity at saturation are given by the equations

$$
\alpha_{P}=\beta_{T} \frac{\mathrm{d} P}{\mathrm{~d} T}-\frac{\mathrm{d} \log \rho}{\mathrm{d} T}
$$

and

$$
C_{p}=C_{s}-\alpha_{P} \frac{\mathrm{d} P}{\mathrm{~d} T} .
$$

Here the density $\rho$, the saturated pressure $P$ and their derivatives with respect to the temperature $T$ are given in DIPPR as explicit analytical formulae with the tabulated coefficients. The same is valid for the saturated heat capacity $C_{s}$.

Therefore, the only required thermodynamic quantity absent in DIPPR is the isothermal compressibility $\beta_{T}$. However, the isothermal compressibility is a quantity, the predictive calculation of which is a sufficiently hard problem not only for various cubic equations of state with adjustable coefficients (RowLinson, 2013) but also for the much more complicated Statistical Associating Fluid Theory (SAFT) equations, which provide accurate results in a single phase region under high pressures but can exhibit large deviations on the coexistence curve (Diamantonis, Economou, 2011).

As a result, some authors argued (LAFITTE et al., 2006; LiANG et al., 2012) that pure volumetric data for 
the coexisting liquid and vapour phases are insufficient to reproduce the speed of sound with an appropriate accuracy.

During the last years, some improvements in the prediction of the saturated speed of sound were reached by the hybridising cubic EoS and SAFT approaches (PolishuK, 2011) or via the usage of the critical data and the density at the triple point, so called CP-PC-SAFT model (PolishuK, 2014). Thus, the basic required parameters of these models can be obtained from the DIPPR reference data only. However, this method requires cumbersome computational procedures.

Another approach, which deals with the volumetric data used as a reference basis, is the usage of phenomenological correlations for the Helmholtz free energy adjusted to the concrete individual liquids. Correspondingly, the speed of sound is searched via its numerical differentiation. There are known methods of multiparametric regression, see the reviews (SPAN et al., 2001; BELL et al., 2014) and references therein as well as even artificial neural networks (LAUGIER, RichON, 2003) trained to reproduce such a kind of the phenomenological equation of state. However, these procedures are also too complex in implementation. In addition, it does not provide a clear physical interpretation of the adjusted parameters.

The main goal of the present work is to propose an extremely simple approach to the calculation of the speed of sound based on the recent results of the studies of the saturated isothermal compressibility (CHORĄżEWSKI et al., 2015), to improve it, and to analyse the consequent usage of DIPPR database data for practically oriented applications.

\section{Method of calculations}

One of the simple universal dimensionless correlations available for the prediction of the isothermal compressibility is the expression proposed in (BRELVI, O'Connell, 1972) for the reduced bulk modulus $\nu=\mu_{0} / R T \rho \beta_{T}$, which has the form

$$
\begin{aligned}
\nu= & \exp \left[-0.42704\left(\xi \rho_{r}-1\right)+2.089(\xi \rho-1)^{2}\right. \\
& \left.-0.42367(\xi \rho-1)^{3}\right]-1,
\end{aligned}
$$

where $\rho_{r}=\rho / \rho_{c}$ is the reduced density ( $\rho_{c}$ is the critical density).

However, it contains the dimensionless factor $\xi$, which is close to unity but its exact value is specific for each substance. Recently a procedure for its determination, which refers to the reduced density $\rho_{r}^{(b)}$ and the reduced bulk modulus $\nu^{b}$ determined at the normal boiling temperature $T_{b}$ has been proposed (CHORĄżEWski et al., 2015):

$$
\xi=1+\frac{a^{*}}{\rho_{r}^{(b)}\left[-5.87605+6.72002 \rho_{r}^{(b)}-1.27101\left(\rho_{r}^{(b)}\right)^{2}\right]},
$$

where

$$
\begin{aligned}
a^{*}= & \log \left(\nu^{b}+1\right)-\left(-0.42704\left(\rho_{r}^{(b)}-1\right)\right. \\
& \left.+2.089\left(\rho_{r}^{(b)}-1\right)^{2}-0.42367\left(\rho_{r}^{(b)}-1\right)^{3}\right) .
\end{aligned}
$$

Thus, the main question is an adequate determination of the reduced bulk modulus in the normal boiling point $\nu^{b}$.

The simultaneous solution of the system formed by Eq. (2) and the definition of the internal pressure $P_{i}$ provided by the thermodynamic equation of state

$$
P_{i}=T \frac{\alpha_{P}}{\beta_{T}}-P
$$

results in the expression for the reduced bulk modulus along the coexistence curve

$$
\nu=\frac{P_{i}}{T}\left[-\frac{R T}{\mu_{0}} \frac{\mathrm{d} \rho}{\mathrm{d} T}\right]^{-1}\left[1-\frac{T}{P_{i}}\left(\frac{\mathrm{d} P}{\mathrm{~d} T}-\frac{P}{T}\right)\right] .
$$

Since the internal pressure is much more larger than the saturated vapour pressure far from the critical point, the expression above can be simplified: the last multiplier can be neglected, and the required reduced bulk modulus at the boiling point takes the form

$$
\nu^{b}=\frac{P_{i}^{b}}{T_{b}}\left[-\left.\frac{R T_{b}}{\mu_{0}} \frac{\mathrm{d} \rho}{\mathrm{d} T}\right|_{T=T_{b}}\right]^{-1} .
$$

As a background, the Trouton-Hildebrand-Everett rule (T-H-E) and its interplay with the internal pressure, see for a comprehensive review (NASH, 1984; MARCUs, 2013), can be used.

It is known that the simplest Hildebrand's correspondence to the latent heat of evaporation $L$,

$$
P_{i L}^{b} \cong \frac{\rho_{b}}{\mu_{0}} L\left(T_{b}\right)
$$

fulfils for non-polar substances only, and requires some compound-dependent small correction factors even in this case.

For this reason, the full T-H-E expression

$$
P_{i T}^{b}=\frac{\rho_{b}}{\mu_{0}}\left(\zeta R+\log T_{b}\right)
$$

seems to be more appropriate due to its wider universality. Here the factor multiplied by $R$ may take its value from the range $\zeta=4.0 \div 4.5$ depending on a symmetry of the intermolecular potential (NASH, 1984). Basing on the evaluated studies, the following correlation of this parameter with the Pitzer acentric factor $\omega$ is proposed:

$$
\zeta=4.44-0.78 \omega
$$


As the third variant, the Srivastava's correlation

$$
P_{i}^{b}=\frac{\rho_{b}}{\mu_{0}} \frac{4.5\left(24.5 T_{b}-1400\right)}{T_{b}}
$$

can be considered. It has been initially derived from the speed of sound measurements (SRIVASTAVA, 1959) and was noted as a good approximation during the studies of the internal pressure (MARCUS, 2013) and as a reference point for the thermodynamic quantities of liquids in a single-phase region (CHORĄŻEWSKI et al., 2015). However, this correlation can be improved comparing Srivastava's and T-H-E equations, which differ in the functions approximating the latent heat. Whence, the factor 4.5 in (10) should be replaced by (9).

This replacement

$$
P_{i S}^{b}=\frac{\rho_{b}}{\mu_{0}} \frac{(4.44-0.78 \omega)\left(24.5 T_{b}-1400\right)}{T_{b}}
$$

provides a better accuracy and will be used further on.

\section{Results}

To test the proposed approach, a set of data for different classes of hydrocarbons with 6 carbon atoms was used: n-hexane (NERUCHEV et al., 2005), n-hexene (Zotov et al., 1975), 1-hexanol (Zotov et al., 1968), cyclohexane (Zotov et al., 1995), cyclohexene (ZoTov et al., 1995), and benzene (NERUCHEV et al., 2005). All these measurements were done in one laboratory, namely, in the Laboratory of Molecular Acoustics at Kursk State University (formerly Kursk State Pedagogical University and Kursk State Pedagogical Institute), which ensures a certain uniformity of the applied methods by providing the equipment and hence, accuracy for this comparative analysis. The data for n-hexane, n-hexanol, and benzene are approved by NIST (National Institute of Standards and Technology) and included into ThermoData Engine (TDE) database. As for the other substances, some other available data for shorter temperature intervals are included into the discussion.

The speed of sound is calculated using Eqs. (1)-(6) for three variants of the internal pressure approximations, Eqs. (7)-(11). Table 1 presents a summary of results related to the prediction accuracy, and Figs. 1-6 allow the discussion of each compound in detail.

Table 1 shows that the accuracy of prediction drastically differs for the temperature interval: it is sufficiently better for $T<T_{b}$. This fact may originate not only from the lower accuracy of Eq. (4) approaching the subcritical region, but also from the less accurate approximation of the heat capacity by the DIPPR polynomial for high temperatures.

Both the first raw in Table 1 and Fig. 1 confirm the known fact that non-polar, non-associating liquid n-hexane satisfies the Hildenrand theory for the enthalpy of evaporation and its interconnection with the
Table 1. AAD (\%) for the calculated values of the speed of sound and the experimental data for the studied liquids along the saturation curve within two ranges separated by the normal boiling temperature $T_{b}$. The letters $\mathrm{S}, \mathrm{T}, \mathrm{L}$ denote the modified Srivastava's, Trouton-Everett's correlations and the usage on the boiling latent heat, correspondingly.

\begin{tabular}{|l|c|c|c|}
\hline \multicolumn{1}{|c|}{$T<T_{b}$} & $S$ & $T$ & $L$ \\
\hline n-hexane & 1.43 & 0.41 & 0.22 \\
1-hexene & 1.67 & 0.74 & 0.57 \\
1-hexanol & 0.35 & 0.80 & 15.0 \\
cyclohexane & 0.43 & 3.06 & 2.94 \\
cyclohexene & 1.14 & 1.67 & 0.85 \\
benzene & 2.08 & 0.79 & 1.58 \\
\hline$T \geq T_{b}$ & $S$ & $T$ & $L$ \\
\hline n-hexane & 1.34 & 2.30 & 1.98 \\
1-hexene & 4.02 & 4.93 & 4.58 \\
1-hexanol & 1.39 & 1.17 & 15.4 \\
cyclohexane & 1.66 & 3.39 & 3.31 \\
cyclohexene & 2.57 & 4.53 & 3.86 \\
benzene & 1.40 & 2.72 & 1.49 \\
\hline
\end{tabular}

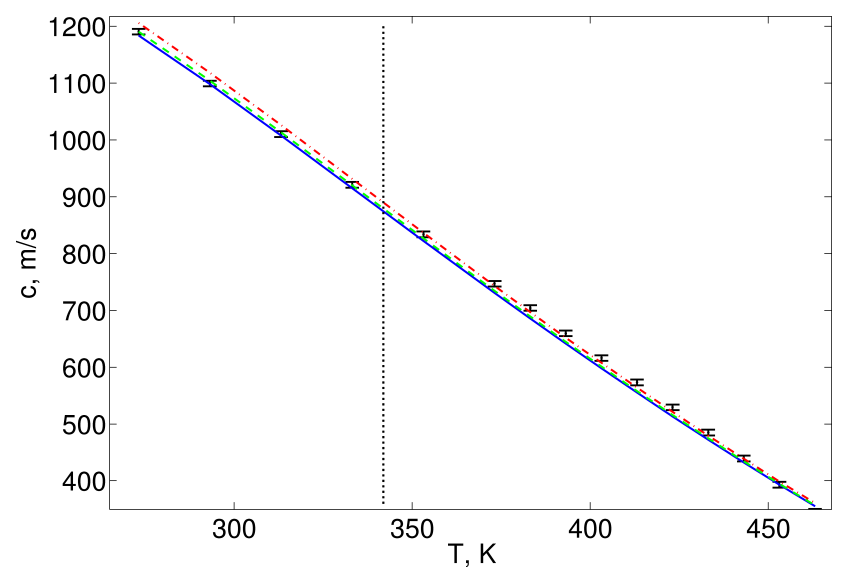

Fig. 1. Speed of sound in n-hexane. The experimental data (NERUCHEv et al., 2005) (dots with error bars indicating $\pm 5 \mathrm{~m} / \mathrm{s}$; this value is larger than the declared error of measurements and provided for visual guiding during the discussion of the DIPPR-based approximation) and their DIPPR-based calculated lines: with the usage (8), solid line; (7), dashed line; (11), dash-dotted line.

cohesive energy and the internal pressure (MARCUs, 2013). The T-H-E approach for the approximation of the internal pressure leads to, in principle, the same accuracy for the prediction of the speed of sound. The overall error in both methods corresponds to the errors of DIPPR correlations: one can see in Fig. 1 that both dashed and solid lines go within errorbars, which are adjusted to the latter.

The correlation (11) results a three times worse than AAD. It is interesting to compare this situation 


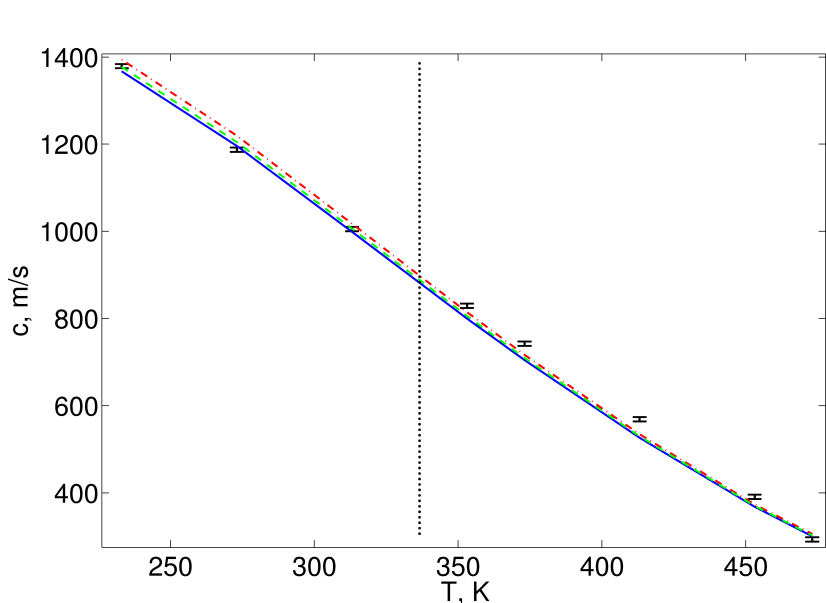

Fig. 2. Speed of sound in 1-hexene. The experimental data (Zotov et al., 1975) (dots with error bars indicating $\pm 5 \mathrm{~m} / \mathrm{s}$; this value is larger than the declared error of measurements and provided for visual guiding during the discussion of the DIPPR-based approximation) and their DIPPR-based calculated lines: with the usage (8), solid line; (7), dashed line; (11), dash-dotted line. The data for the speed of sound measured at the normal pressure (KHASANSHIN et al., 2005) are shown as circles for comparison.

with the results for 1-hexanols, i.e. a typical polar liquid, see Fig. 3. Naturally, the speed of sound calculated via (7) fails completely in this case. In contrast, the Srivastava-based internal pressure leads to the best result, $A A D=0.35 \%$. However, and T-H-E-based curve goes within the errorbars for the experimental points $T<T_{b}$ except for the lowest $T=293 \mathrm{~K}$. But even the presence of this point does not allow AAD to exceed $0.8 \%$.

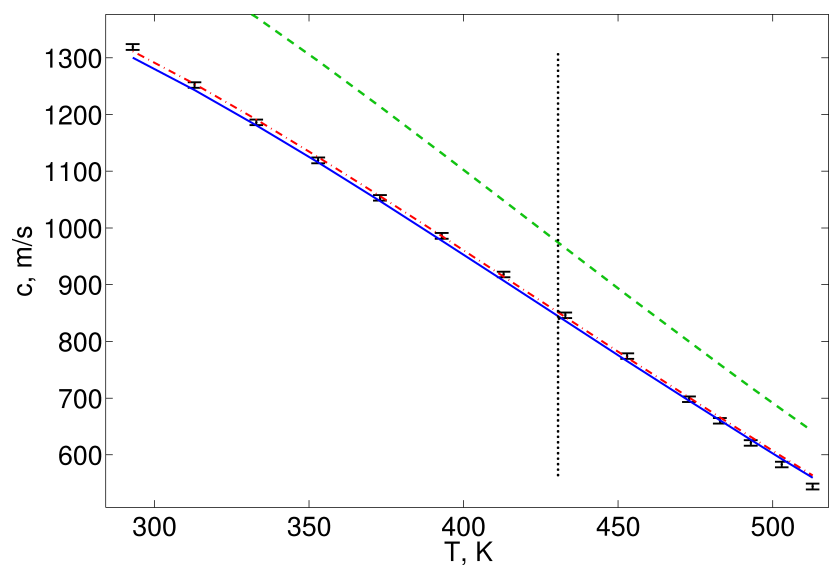

Fig. 3. Speed of sound in 1-hexanol. The experimental data (NERUCHEV et al., 2005) (dots with error bars indicating $\pm 5 \mathrm{~m} / \mathrm{s}$; this value is larger than the declared error of measurements and provided for visual guiding during the discussion of the DIPPR-based approximation) and their DIPPR-based calculated lines: with the usage (8), solid line; (7), dashed line; (11), dash-dotted line.

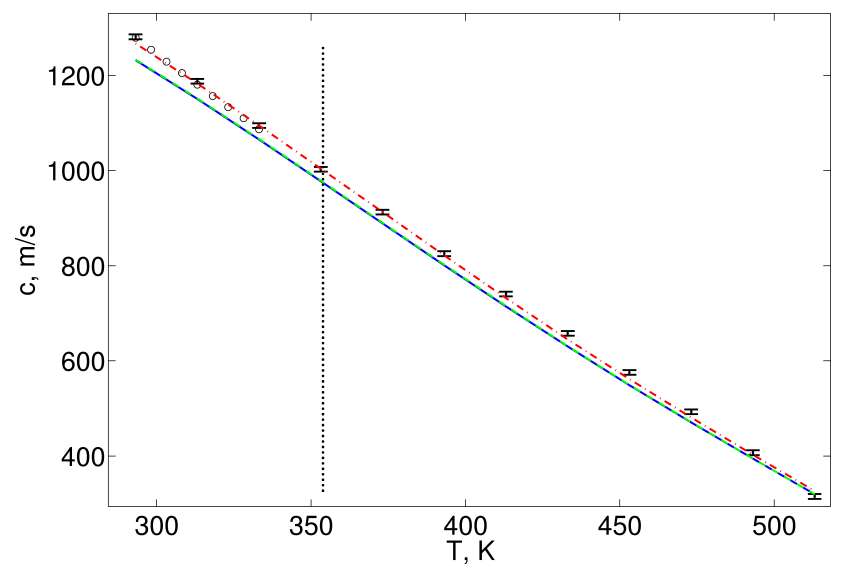

Fig. 4. Speed of sound in cyclohexane. The experimental data (Zotov et al., 1995) (dots with error bars indicating $\pm 5 \mathrm{~m} / \mathrm{s}$; this value is larger than the declared error of measurements and provided for visual guiding during the discussion of the DIPPR-based approximation) and their DIPPR-based calculated lines: with the usage (8), solid line; (7), dashed line; (11), dash-dotted line. The data for the speed of sound measured at the normal pressure (CERDEIRIÑ et al., 2001) are shown as circles for comparison.

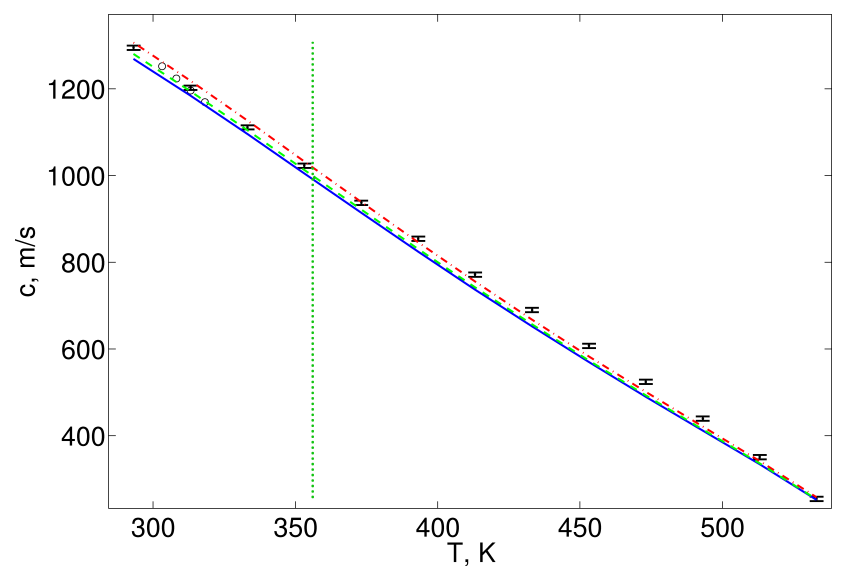

Fig. 5. Speed of sound in cyclohexene. The experimental data (ZoTov et al., 1995) (dots with error bars indicating $\pm 5 \mathrm{~m} / \mathrm{s}$; this value is larger than the declared error of measurements and provided for visual guiding during the discussion of the DIPPR-based approximation) and their DIPPRbased calculated lines: with the usage (8), solid line; (7), dashed line; (11), dash-dotted line. The data for the speed of sound measured at the normal pressure (REDDY et al., 2014) are shown as circles for comparison.

The same behaviour with respect to (8)-(9) should be noted for benzene too. The main source of the overall error is the interval $T<300 \mathrm{~K}$, all other predictions for $300 \mathrm{~K}<T<T_{b}$ practically coincide with the experimental points, see Fig. 6. Benzene is a nonpolar liquid, but it can form dimer associates. Whence, Eq. (11) gives a large error too.

Three other liquids are significantly less explored with respect to the speed of sound along the saturation 


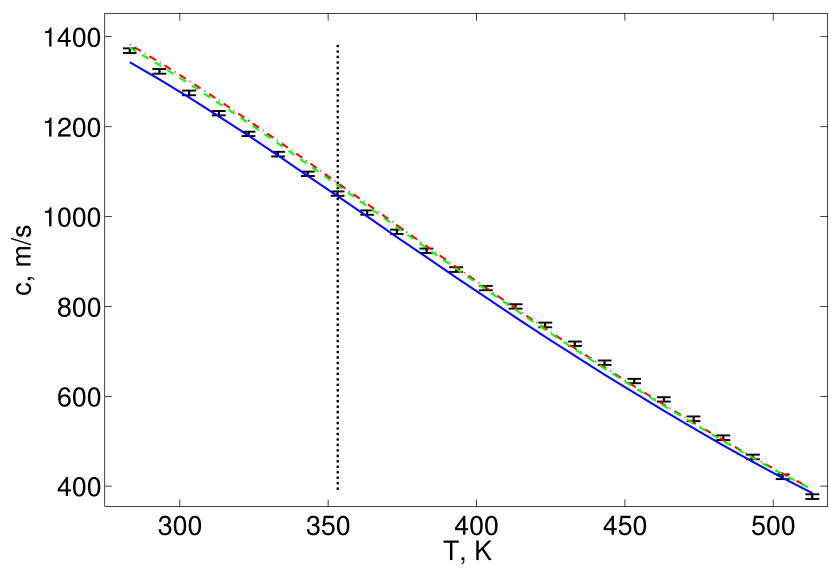

Fig. 6. Speed of sound in benzene. The experimental data (NeRuChev et al., 2005) (dots with error bars indicating $\pm 5 \mathrm{~m} / \mathrm{s}$; this value is larger than the declared error of measurements and provided for visual guiding during the discussion of the DIPPR-based approximation) and their DIPPR-based calculated lines: with the usage (8), solid line;

(7), dashed line; (11), dash-dotted line.

line. For this reason, the comparison with some other data obtained at the normal pressure for the temperatures below the boiling point is added. The variation of these experimental data consists of $0.5-1 \%$. However, the T-H-E-based prediction, as well as the Hildebrandbased one, is less than $1 \%$ for both sets of data (for the experimental data taken from (KHASANSHIN et al., 2005) the triplet of AAD is $(1.66,0.6,0.86)$, i.e., slightly better for (8)-(9) and slightly worse for (11)).

The rest two liquids, cyclohexane and cyclohexene, deviate from the described regularity. They both have cyclic molecules. However, in contrast to benzene, the best correlation is found for the usage of (11) for cyclohexane and (7) for cyclohexene, but not for the T-H-E relation. Two additional short sets of data for $T<T_{b}$ give the triplets of AAD: $(0.48,2.68,2.55)$ for (CERDEIRIÑA et al., 2001), and $(1.60,1.26,0.54)$ for (REDDY et al., 2014). All these observations show that the speed of sound can be predicted using DIPPR data for cyclohexane and cyclohexene but the reason for the proper choice of the internal pressure equation needs some further investigations. An additional warning in this case is the limited number of experimental data for these substances (especially, in the wide region of state parameters, e.g., close to the boiling point and within the interval between it and the critical point), which, in addition deviated somewhere up to $1 \%$. Thus, a further discussion requires new extensive measurements of the speed of sound with a higher precision.

As for the region $T>T_{b}$, the accuracy of approximation is $2 \div 5$ time worse, and such a behaviour is noted for all studied compounds. This fact can originate not only from the less accurate fitting of the reduced bulk modulus by Eq. (4) but also from the less accurate DIPPR correlations for the heat capacity.

At the same time, the substances considered above have relatively close values of their molecular weights, although their molecular structures are sufficiently different. For this reason, an additional test was carried out with respect to the carbon chain's length. Since the basic used correlation (4) operates on the reduced density, Fig. 7 represents the data with respect to this variable. The experimental data for n-hexane, noctane, n-dodecane, n-hexadecane, and n-tetracosane are taken from (NERUCHEV et al., 2005), and those for n-squalane are taken from (KorotKovski et al., 2012).
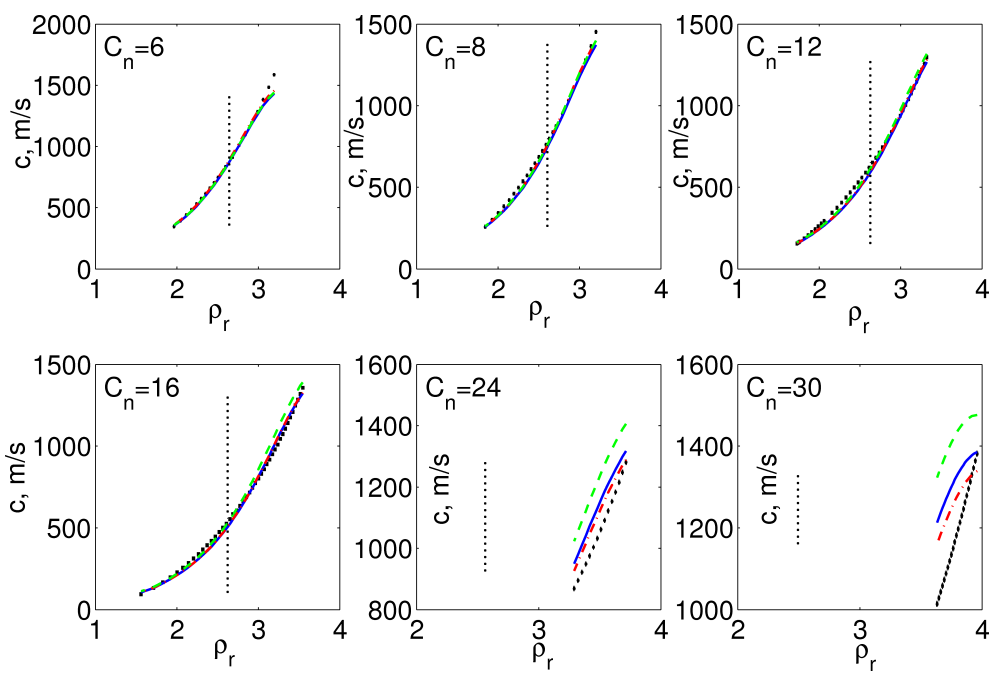

Fig. 7. Speed of sound in alkanes with different lengths of carbon chain denoted as $C_{n}$ represented as a function of the reduced density $\rho / \rho_{r}$. All line notations for the experimental and calculated data are the same as in Figs. 1-6. The vertical dashed line provides a visual reference to the location of the reduced density corresponding to the boiling point. 
This figure indicates that the proposed methods work correctly for n-alkanes up to $\mathrm{C}_{16} \mathrm{H}_{34}$ : the AAD does not exceed $1 \%$ for the internal pressure correlation (8) for all these substances for the reduced densities corresponding to the temperatures larger than the boiling temperature. Equation (11) gives similar results. Three points which are exceptions for n-hexane and one point for n-octane correspond to the region of low temperatures and high speeds of sound which are close to the melting point.

At the same time, the error starts to increase for longer chains. The predicted results have a very significant deviation (5.4\% and 8.9\% AAD for (11) and (8), respectively) for $\mathrm{C}_{24} \mathrm{H}_{50}$ and are completely inadequate for $\mathrm{C}_{30} \mathrm{H}_{62}$.

The origin of such a behaviour can be revealed inspecting the plot of the Brelvi-O'Connel correlation (4) for the reduced bulk modulus, see Fig. 8. One can see that the function grows (which is physically correct) up to $\rho_{r}=4.18$, and decays further on. The decay corresponds to a non-physical growth of the compressibility. Thus, the predicted values for $\rho_{r}>4.18$ should be rejected. Moreover, the slowing growth for $3.4 \div 3.5<\rho_{r}<4.18$ is the source of significant errors as well. Figure 7 shows that incorrect predictions fall into this interval. Otherwise, the Brelvi-O'Connell correlation provides quite accurate results (note that its numerical parameters were originally adjusted in the case of such moderate reduced densities (BRELVI, O’CONnell, 1972).

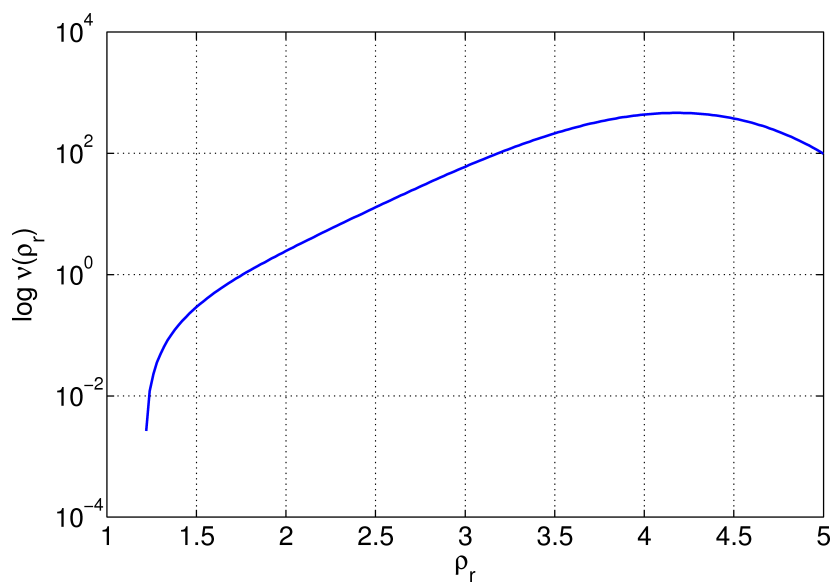

Fig. 8. Function of Brelvi-O'Connell representing the reduced bulk modulus as a function of the reduced density for $\xi=1$.

\section{Conclusion}

It is shown that DIPPR equations (defining standard references for the density, pressure, and heat capacity at the saturation conditions), accompanied by the Brelvi-O'Connell correlation for the reduced bulk modulus, provide an opportunity to obtain the saturated speed of sound for the temperatures lower than the boiling point and not too close to the freezing conditions. The accuracy of such an approximation corresponds to the accuracy of the rest of thermodynamic values in the DIPPR database if the proper correlation is chosen for the internal pressure at the boiling point.

Among the available correlations, the most sure is the expression (8) based on the Trouton-HildebrandEverett rule and the proposed correlation with the acentric factor (9). Although other studied correlations can give a better accuracy for some studied substances, the T-H-E-based approach is most stable and, on average, gives results comparable with the overall DIPPR database errors of estimation.

At the same time, the proposed approach has some restrictions on its area of applicability: (i) due to the analysed properties of the Brelvi-O'Connell correlation, one can use it for the thermodynamic states corresponding to the reduced densities smaller than $\rho_{r}=3.5$; (ii) the guaranteed predicted speeds of sound are bounded by the values smaller than $c=1500 \mathrm{~m} / \mathrm{s}$. These conditions provide a direction to future studies of the reduced bulk modulus correlations applicable for a wider region of thermodynamic parameters.

\section{Acknowledgment}

The work is supported by RFBR, research project No. 16-08-01203.

\section{References}

1. Aspen Plus, http://www.aspentech.com/products/engineering/aspen-plus/

2. Bell I.H., Wronski J., Quoilin S., Lemort V. (2014), Pure and pseudo-pure fluid thermophysical property evaluation and the open-source thermophysical property library CoolProp, Industrial \& Engineering Chemistry Research, 53, 2498-2508.

3. Brelvi S.W., O'Connell J.P. (1972), Corresponding states correlations for liquid compressibility and partial molal volumes of gases at infinite dilution in liquids, AIChE Journal, 18, 1239-1243.

4. Cerdeiriña C.A., Tovar C.A., GonzÁlez-SalgaDo D., Carballo E., Romani L. (2001), Isobaric thermal expansivity and thermophysical characterization of liquids and liquid mixtures, Physical Chemistry Chemical Physics, 3, 5230-5236.

5. Chorążewski M., Postnikov E.B., Oster K., Polishuk I. (2015), Thermodynamic Properties of 1 , 2-Dichloroethane and 1, 2-Dibromoethane under Elevated Pressures: Experimental Results and Predictions of a Novel DIPPR-Based Version of FT-EoS, PC$S A F T$, and $C P-P C-S A F T$, Industrial \& Engineering Chemistry Research, 54, 9645-9656.

6. Diamantonis N.I., Economou I.G. (2011), Evaluation of statistical associating fluid theory (SAFT) and perturbed chain-SAFT equations of state for the calculation of thermodynamic derivative properties of fluids 
related to carbon capture and sequestration, Energy \& Fuels, 25, 3334-3343.

7. DIPPR 801 Database, http://www.aiche.org/dippr/ events-products/801-database

8. Khasanshin T.S, Poddubskit O.G., ShchemeLEV A.P. (2005), Sound Velocity in Liquid 1-Alkenes, High Temperature, 43, 530-537.

9. Korotkovskit V.I., Lebedev A.V., Ryshkova O.S., Bolotnikov M.F., Shevchenko Y.E., NeruCHEV Y.A. (2012), Thermophysical properties of liquid squalane $\mathrm{C}_{30} \mathrm{H}_{62}$ within the temperature range of 298.15-413.15 K at atmospheric pressure, High Temperature, 50, 471-474.

10. Lafitte T., Bessieres D., Piñeiro M.M., DariDON J.L. (2006). Simultaneous estimation of phase behavior and second-derivative properties using the statistical associating fluid theory with variable range approach Journal of Chemical Physics, 124, 024509.

11. Laugier S.D., Richon D. (2003), Use of artificial neural networks for calculating derived thermodynamic quantities from volumetric property data /Fluid phase equilibria, 210, 247-255.

12. Liang X., Maribo-Mogensen B., Thomsen K., Yan W., Kontogeorgis G.M. (2012), Approach to improve speed of sound calculation within PC-SAFT framework, Industrial \& Engineering Chemistry Research, 51, 14903-14914.

13. Marcus Y. (2013), Internal pressure of liquids and solutions, Chemical Reviews, 113, 6536-6551.

14. NASH L.K. (1984), Trouton and T-H-E rule, Journal of Chemical Education, 61 981-984.

15. Neruchev Y.A., Bolotnikov M.F., Zotov V.V. (2005), Investigation of ultrasonic velocity in organic liquids on the saturation curve, High temperature, 43, 266-309.

16. Polishuk I. (2011), Hybridizing SAFT and cubic EOS: what can be achieved? Industrial \& Engineering Chemistry Research, 50, 4183-4198.
17. Polishuk I. (2014), Standardized critical point-based numerical solution of statistical association fluid theory parameters: the perturbed chain-statistical association fluid theory equation of state revisited, Industrial \& Engineering Chemistry Research, 53, 14127-14141.

18. Reddy K.R., Kumar D.B.K., Rao G.S., Anila P., Rambabu C. (2014), Densities, viscosities, sound speed, and IR studies of $N$-methyl-2-pyrrolidone with cyclohexylamine, cyclohexanol, and cyclohexene at different temperatures, Thermochimica Acta, 590, 116126.

19. Rowlinson J.S., Swinton F.L. (2013), Liquids and Liquid Mixtures, Butterworth-Heinemann.

20. Sandler S.I. (2015), Using Aspen Plus in thermodynamics instruction: a step-by-step guide, John Wiley \& Sons.

21. Span R., Wagner W., Lemmon E.W., JacobSEN R.T. (2001), Multiparameter equations of staterecent trends and future challenges, Fluid Phase Equilibria, 183, 1-20.

22. SRIVAStava S.C. (1959), Relationship between ultrasound velocity and other physical properties of pure organic liquids, Indian Journal of Physics, 33, 503-504.

23. Zotov V.V., Kireev B.N., Neruchev Y.A. (1975), Study of the equilibrium properties of hydrocarbons on the saturation line by an acoustic method, Journal of Applied Mechanics and Technical Physics, 16, 282284.

24. Zotov V.V., Melikhov Y.F., Mel'nikov G.A., NerucheV Y.A. (1995), Sound Velocity in Liquid Hydrocarbons [in Russian], Kursk State Pedagog. Univ., Kursk.

25. Zotov V.V., Neruchev Y.A., OtpushchenniKOV N.F. (1968), Speed of sound on the saturation line for some monohydric aliphatic alcohols, Russian Physics Journal, 11, 130-131. 